\section{Screening for diabetic retinopathy using digital colour photography and oral fluorescein angiography}

\begin{abstract}
Purpose To evaluate digital colour photography and oral fluorescein angiography (OFA) for diabetic retinopathy screening. Methods Thirty-seven patients were selected from either a diabetic retinopathy screening or a medical retina clinic. Three $45^{\circ}$ colour digital images and a single macula $45^{\circ}$ OFA image were taken from each eye. Standard sevenfield stereo photography with ETDRS grading was used as a gold standard for data comparison. The images were assessed by two graders and the results of each method compared using the McNemar test.

Results Five eyes had no diabetic retinopathy, 50 had background diabetic retinopathy, 3 had pre-proliferative diabetic retinopathy, 11 had proliferative disease and 3 had quiescent posttreatment disease. Clinically significant macular oedema was present in 25 eyes and absent in 48. For grading diabetic retinopathy digital colour photography produced a sensitivity of 0.87 (specificity 0.83 ); OFA produced a sensitivity of 0.87 (specificity 0.80 ) $(p=0.1)$. For the detection of diabetic maculopathy, the sensitivity of digital colour photography was 0.48 (specificity of 0.95 ) and for OFA was 0.87 (specificity 0.87$)(p<0.01)$. Conclusion This pilot study has shown that both digital colour photography and OFA compare well with conventional methods for diabetic retinopathy screening. The results encourage the further evaluation of OFA in the screening for diabetic maculopathy.
\end{abstract}

Key words Diabetic retinopathy, Digital photography, Oral fluorescein angiography, Screening

Screening for diabetic retinopathy is good clinical practice and cost-effective health care. ${ }^{1-5}$ Screening methods vary widely and they have been extensively assessed. ${ }^{6-8}$ The use of direct ophthalmoscopy is now recognised as a poor method and photography or biomicroscopy are
RICHARD NEWSOM, BEN MOATE, TONY CASSWELL advocated. Photography has the advantage that permanent retinal images are produced allowing for rapid diagnosis and also audit/ quality assesment.

We appraised two new photographic methods for diabetic retinopathy screening: digital colour photography and oral fluorescein angiography (OFA). Digital colour photography was chosen as it is replacing conventional colour photography. ${ }^{9}$ This technique gives highdefinition images which are easily stored on, and enhanced by, computers. Digital images may also be used for automated diabetic retinopathy detection using artificial neural networks. ${ }^{10}$ The other test was OFA, imaged with a digital camera. Intravenous (i.v.) fluorescein angiography is a sensitive technique for the detection of early diabetic retinopathy. ${ }^{11}$ However, fluorescein need not be administered intravenously. OFA has been performed for many years and is currently used for children and in patients with no intravenous access. ${ }^{12-14}$ It benefits from being non-invasive and having fewer side effects than the i.v. method, ${ }^{15}$ although there is a case report of allergic reaction following OFA. ${ }^{16}$

OFA has not been widely used because it produces poorer quality images than i.v. angiography. The OFA images have less contrast (due to a lower plasma concentration of fluorescein ${ }^{17}$ ) and OFA does not produce earlyphase angiogram images. However, early-phase shots are not necessary for the detection of many conditions such as diabetic maculopathy, neovascularisation and papilloedema. ${ }^{18}$

The development of digital cameras has overcome some of the drawbacks of OFA. These cameras are more light-sensitive, so lower levels of fluorescence can be detected, and the images may be digitally enhanced giving good final results. These considerations encouraged us to re-evaluate OFA as a method of detecting retinal disease.

This study aimed to assess the feasibility of using colour digital photography and OFA to detect sight-threatening diabetic retinopathy.
R. Newsom

B. Moate

A. Casswell

Sussex Eye Hospital Brighton, UK

Mr R.S.B. Newsom Moorfields Eye Hospital City Road London EC1V 2PD, UK e-mail:

rbnewsom@dircon.co.uk

Received: 18 March 1999 Accepted in revised form: 11 February 2000 


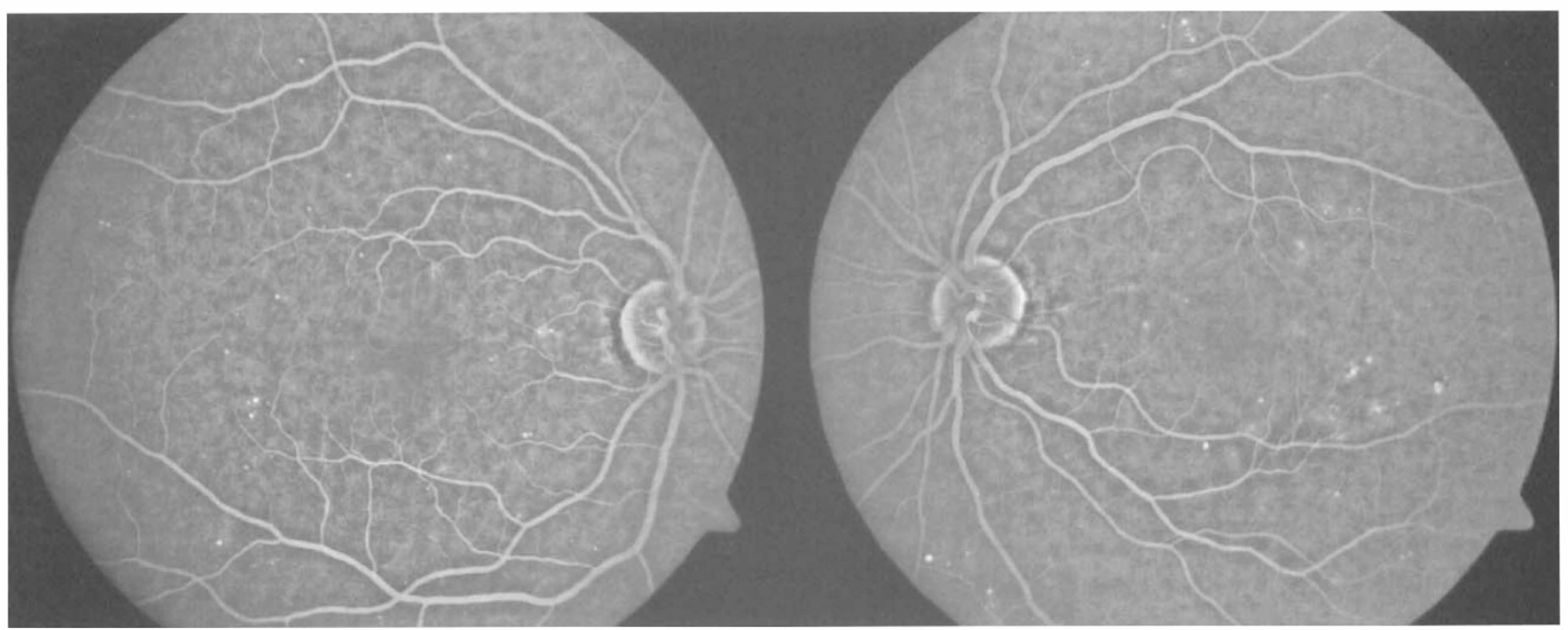

Fig. 1. An enhanced oral fluorescein angiogram, demonstrating the reasonable quality and high contrast that can now be afforded by this technique.

\section{Methods}

Thirty-seven patients were recruited from a diabetic retinopathy clinic and a diabetic screening service. After explanation and consent the patients were dilated with $1 \%$ tropicamide. They were given a dose of fluorescein sodium (between 1 and $2 \mathrm{mg} / \mathrm{kg}$ ) orally which was diluted in $250 \mathrm{ml}$ of orange juice. Blood pressure and pulse were monitored by the trial nurse. Thirty minutes later, three $45^{\circ}$ digital colour photographs were taken with a Zeiss FF450 camera (Carl Zeiss, UK), corresponding to ETDRS grading fields F1, F4 and F5. ${ }^{19}$ A single $45^{\circ}$ macula angiogram image was taken at $35-40$ min (Fig. 1). The patients were then transferred onto a standard $30^{\circ}$ retinal camera for seven-field stereo photography. Each patient gave informed consent for retinal photography and OFA, and the trial was performed under the auspices of the local ethics committee.

The digital images were graded by two ophthalmologists, and the seven-field stereo photographs by an ETDRS trained grader as the gold standard. Images were graded as: (i) no retinopathy, (ii) background retinopathy, (iii) pre-proliferative retinopathy (iv) proliferative retinopathy and (v) posttreatment group. A separate grade was given for the presence or absence of clinically significant macular oedema (ETDRS). ${ }^{19}$

Table 1. Digital colour photography grading of diabetic retinopathy

\begin{tabular}{lccc}
\hline & True positive & True negative & Total \\
\hline Test positive & 53 & 1 & 54 \\
Test negative & 8 & 5 & 13 \\
Total & 61 & 6 & 67 \\
\hline
\end{tabular}

Sensitivity $=0.87$, specificity $=0.83, \mathrm{PPV}=0.98, \mathrm{NPV}=0.83$. Ungradable images $5 / 72(6.9 \%)$.

\section{Statistical methods}

The sensitivities, specificities, positive predictive value (PPV) and negative predictive values (NPV) were calculated for each test. The proportion of true positives versus false positives for each test were compared using McNemar's test. Statistical advice was obtained from the Department of Statistics, Kings College Hospital, London.

\section{Results}

Thirty-seven patients were recruited (age range 25-70 years). Two of the gold standards were ungradable: one because of inadequate field capture and the other because of media opacities. The gold standard with poor field capture was still unusable for assessment of macular oedema. Of the other 72 eyes, 5 had no diabetic retinopathy, 50 had background diabetic retinopathy, 3 had pre-proliferative diabetic retinopathy, 11 had proliferative disease and 3 had quiescent (posttreatment) disease, 25 had diabetic maculopathy and 48 had no maculopathy.

During the test 3 patients complained of a bitter taste following the fluorescein administration; however, no patient vomited, complained of nausea or suffered any allergic symptoms.

The grading results (for 72 eyes) were compared with seven-field stereo photography for both the grade of retinopathy and the presence or absence of clinically significant diabetic maculopathy. Five of the colour

Table 2. Oral fluorescein angiography grading of diabetic retinopathy

\begin{tabular}{lccc}
\hline & True positive & True negative & Total \\
\hline Test positive & 54 & 1 & 55 \\
Test negative & 8 & 4 & 12 \\
Total & 62 & 5 & 67 \\
\hline
\end{tabular}

Sensitivity $=0.87$, specificity $=0.80, \mathrm{PPV}=0.98, \mathrm{NPV}=0.50$. Ungradable images $5 / 72(6.9 \%)$. Inter-test McNemar $=2.17(p>0.1)$. 
Table 3. Digital colour photography detection of diabetic macular oedema

\begin{tabular}{lccc}
\hline & True positive & True negative & Total \\
\hline Test positive & 12 & 2 & 14 \\
Test negative & 13 & 41 & 54 \\
Total & 25 & 43 & 68 \\
\hline
\end{tabular}

Sensitivity $=0.48$, specificity $=0.95, \mathrm{PPV}=0.86, \mathrm{NPV}=0.95$.

Ungradable images $5 / 72(6.9 \%)$.

Table 4. Oral fluorescein angiography detection of diabetic macular oedema

\begin{tabular}{lccc}
\hline & True positive & True negative & Total \\
\hline Test positive & 20 & 6 & 26 \\
Test negative & 3 & 39 & 42 \\
Total & 23 & 45 & 68 \\
\hline
\end{tabular}

Sensitivity $=0.87$, specificity $=0.87, \mathrm{PPV}=0.77, \mathrm{NPV}=0.87$.

Ungradable images $5 / 72(6.9 \%)$.

Inter-test McNemar $=10.32(p<0.01)$.

digital images and 5 of the oral fluorescein angiograms were ungradable. The results are presented on 67 colour digital gradings and fluorescein angiograms for the grading of retinopathy and on 68 images for the detection of macular oedema. The results are shown in Tables 1-4.

Table 1 shows the results for the grading of diabetic retinopathy using digital colour photography: a sensitivity of 0.87 with a specificity of 0.83 . The result for OFA was comparable (Table 2), with a sensitivity of 0.87 and a specificity of 0.80 ; the inter-test McNemar result was $2.17(p>0.1)$. When digital colour photography was used to assess macular oedema (Table 3 ) a sensitivity of 0.48 (specificity of 0.95 ) was found. In comparison OFA (Table 4$)$ was more sensitive $(0.87$; specificity 0.87$)$, the inter-test McNemar result being $10.32(p<0.01)$.

\section{Discussion}

We have demonstrated that digital OFA and digital colour photography are sensitive methods for the detection of diabetic retinopathy. Digital colour photography yielded a sensitivity of 0.87 (PPV 0.98) and OFA a sensitivity of 0.87 (PPV 0.99). These compare well with the previously published data using $35 \mathrm{~mm}$ colour photography and Polaroid photography. Moss et al. ${ }^{20}$ showed that four-field stereo $30^{\circ}$ images have a sensitivity of $89 \%$ for the detection of diabetic retinopathy. Others have further simplified this method by using a three-field non-stereo pattern ${ }^{21}$ and maintained a high of sensitivity of $89 \%{ }^{22}$ These results reflect the best in conventional photography, using highdefinition $35 \mathrm{~mm}$ film. Kerr et al. ${ }^{23}$ found the sensitivity of digital colour photography to be $90 \%$ when compared with a clinician and now use it routinely to follow up patients with established or treated diabetic retinopathy ( $26 \%$ of their clinic). Our results with digital colour photography are in broad agreement (sensitivity of 0.85 ); however, we used seven-field stereo as a gold standard, which is generally more sensitive for detecting diabetic retinopathy.

\section{Detection of diabetic maculopathy}

The best method of screening for macular oedema is still unclear. Many screening programmes use non-stereo retinal images, which may underestimate the incidence of diabetic macular oedema. Some programmes rely on a reduction of acuity to trigger referral for macular oedema. $^{21}$ This is unfortunate as the ETDRS clearly showed that only a minority of patients treated with macular grid experienced an improvement in vision; most patients experienced a stabilisation or moderate acuity loss. Patients referred late for treatment did worse. Conversely, patients with little or no visual loss still benefited from laser treatment compared with those with deferred treatment. ${ }^{24}$ We found non-stereo colour digital images were relatively insensitive for detecting diabetic macular oedema (sensitivity of 0.48 ). OFA, however, yielded a sensitivity of 0.87 . Few studies have reported rates for the detection of diabetic macular oedema. Using slit-lamp biomicroscopy the ETDRS reported that macular oedema was detected $82 \%$ of the time with a specificity of $79 \%$ when compared with stereo photography. This is similar to the sensitivity of OFA.

\section{Photographic failures}

Several techniques have problems with photographic failures. Klein et al. ${ }^{25}$ found that $50^{\circ}$ non-mydriatic cameras had an $86 \%$ sensitivity compared with the gold standard; however, the number of photographic failures increased from $2 \%$ to $12.7 \%$. In our group photographic failure occurred in $2.8 \%$ of patients undergoing $30^{\circ}$ colour photography, compared with $6.9 \%$ for $45^{\circ}$ digital colour images and $6.9 \%$ of OFA images. This failure rate is similar to other methods such as Polaroid photography. $^{26}$

\section{Conclusion}

We have shown, in this pilot study, that digital colour photography and oral fluorescein angiography are highly sensitive for the detection of diabetic retinopathy. However, non-stereo digital colour images are relatively insensitive for detecting clinically significant diabetic maculopathy. Oral fluorescein angiography was as effective as clinical examination in the detection of maculopathy. As early detection of maculopathy is key for preserving vision in these patients, these results highlight the need for a two-stage screening process where patients with a suspicious macula could be referred for further assessment.

We thank Gerry Heath from Carl Zeiss UK for loan of a digital camera. 


\section{References}

1. American Academy of Ophthalmology, American Medical Association, American Diabetic Association. Guidelines for screening for diabetic retinopathy. Ann Intern Med 1992;116:683-5.

2. A protocol for screening for diabetic retinopathy in Europe Retinopathy Working Party. Diabet Med 1991;8(3):263-7.

3. Burns-Cox J. Prevention of blindness: a lost opportunity [editorial]. J Med Screening 1996;3:169.

4. Kohner EM, Porta M. Protocols for screening and treatment of diabetic retinopathy in Europe. Eur J Ophthalmol 1991;1:45-54.

5. Javitt JC, Aiello LP, Chiang Y, Ferris FL 3rd, Canner JK, Greenfield S. Preventive eye care in people with diabetes is cost-saving to the federal government: implications for health-care reform. Diabetes Care 1994;17:909-17.

6. Kalm H, Egertsen R, Blohme G. Non-stereo fundus photography as a screening procedure for diabetic retinopathy among patients with type II diabetes: compared with $60 \mathrm{D}$ enhanced slit-lamp examination. Acta Ophthalmol (Copenh) 1989;67:546-53.

7. Cathelineau G, Cathelineau BV. Diabetic retinopathy: methodologies in practice [review]. Diabet Med 1991;8:S17-22.

8. Davies R, Sullivan P, Canning C. Simulation of diabetic eye disease to compare screening policies. $\mathrm{Br} \mathrm{J}$ Ophthalmol 1996;80:945-50

9. Gardner GG, Keating D, Williamson TH, Elliott AT. Automatic detection of diabetic retinopathy using an artificial neural network: a screening tool [see comments]. Br J Ophthalmol 1996;80:940-4.

10. Undrill $\mathrm{P}$. Towards the interpretation of retinal images. Br J Ophthalmol 1996;80:937-8.

11. Diabetes Control and Complications Trial Research Group. Color photography versus fluorescein angiography in the diabetes control trial. Arch Ophthalmol 1987;105:1344-51.

12. Noble MJ, Cheng H, Jacobs PM. Oral fluorescein and cystoid macular oedema: detection in aphakic and pseudophakic eyes. Br J Ophthalmol 1984;68:221-4.

13. Nayak BK, Ghose S. A method for fundus evaluation in children with oral fluorescein. Br J Ophthalmol 1987;71:907-9.

14. Morgan KS, Franklin RM. Oral fluorescein angioscopy in aphakic children. J Pediatr Ophthalmol Strabismus 1984;21:33-6.
15. Kelley JS, Kincaid M, Hoover RE, McBeth C. Retinal fluorograms using oral fluorescein. Ophthalmology 1980;87:805-11.

16. Kinsella FP, Mooney DJ. Anaphylaxis following oral fluorescein angiography. Am J Ophthalmol 1988;106:745-6.

17. Gomez-Ulla F, Malvar A, Parafita M, Polo P, Seoane I. Oral fluorescein angiography and fluoroscopy: determination of plasma fluorescein levels and clinical application. Optometry Vis Sci 1992;69:986-90.

18. Ghose S, Nayak BK. Role of oral fluorescein in the diagnosis of early papilloedema in children. Br J Ophthalmol 1987;71:910-5.

19. Early Treatment Diabetic Retinopathy Study Group. A modification of the Airlie House classification of diabetic retinopathy. Diabetic Retinopathy Study Report no. 7. Invest Ophthalmol Vis Sci 1981;21:210.

20. Moss S, Meuer S, Klein R, Hubbard L, Brothers R, Klein B. Are seven standard photographic fields necessary for classification of diabetic retinopathy? Invest Ophthalmol Vis Sci 1989;30:823-8.

21. Harding SP, Broadbent DM, Neoh C, White MC, Vora J. Sensitivity and specificity of photography and direct ophthalmoscopy in screening for sight threatening eye disease: the Liverpool Diabetic Eye Study [see comments]. BMJ 1995;311:1131-5.

22. Leese GP, Newton RW, Jung RT, Haining W, Ellingford A. Screening for diabetic retinopathy in a widely spaced population using non-mydriatic fundus photography in a mobile unit. Tayside Mobile Eye Screening Unit. Diabet Med 1992;9:459-62.

23. Kerr D, Cavan DA, Jennings B, Dunnington C, Gold D, Crick $M$. Beyond retinal screening: digital imaging in the assessment and follow up of patients with diabetic retinopathy. Diabet Med 1998;15:878-82.

24. Early Treatment for Diabetic Retinopathy Study Group. Study 1 . Photocoagulation for diabetic macular oedema. Arch Ophthalmol 1985;103:1796-806.

25. Klein R, Klein B, Neider M, Hubbard L, Meuer S, Brothers R. Diabetic retinopathy as detected using ophthalmoscopy, a nonmydriatic camera and a standard fundus camera. Ophthalmology 1985;92:485-91.

26. Ryder R, Young S, Vora J, Atiea J, Owens J, et al. Screening for diabetic retinopathy using poloroid retinal photography through undilated pupils. Practical Diabetes 1985;2:34-9. 